\title{
Programming the Home and Enterprise WiFi with OpenSDWN
}

\author{
Julius Schulz-Zander \\ TU Berlin \\ Berlin, Germany \\ julius@inet.tu-berlin.de \\ Stefan Schmid \\ Deutsche Telekom Innovation \\ Laboratories / TU Berlin \\ Berlin, Germany \\ stefan@inet.tu-berlin.de
}

\author{
Carlos Mayer \\ TU Berlin \\ Berlin, Germany \\ carlosnmayer@gmail.com \\ Anja Feldmann \\ TU Berlin \\ Berlin, Germany \\ anja@inet.tu-berlin.de
}

\author{
Bogdan Ciobotaru \\ TU Berlin \\ Berlin, Germany \\ bogdan.ciobotaru1@gmail.com \\ Roberto Riggio \\ CREATE-NET \\ Trento, Italy \\ rriggio@create-net.org
}

\begin{abstract}
The quickly growing demand for wireless networks and the numerous application-specific requirements stand in stark contrast to today's inflexible management and operation of WiFi networks. In this paper, we present and evaluate OPENSDWN, a novel WiFi architecture based on an SDN/NFV approach. OPENSDWN exploits datapath programmability to enable service differentiation and fine-grained transmission control, facilitating the prioritization of critical applications. OPENSDWN implements per-client virtual access points and per-client virtual middleboxes, to render network functions more flexible and support mobility and seamless migration. OPENSDWN can also be used to out-source the control over the home network to a participatory interface or to an Internet Service Provider.
\end{abstract}

\section{Categories and Subject Descriptors}

C.2.3 [Computer Communication Networks]: Network Operations; C.2.1 [Computer Communication Networks]: Network Architecture and Design

\section{Keywords}

Network Function Virtualization; Software-Defined Networking; Programmable RAN; Enterprise WLANs; Wi-Fi

\section{INTRODUCTION}

In this demo, we show the potential and benefits of introducing programmability and virtualization in wireless networks. Wireless networks are very different from wired networks-the domain where SDN/NFV has been studied most intensively so far-as communication happens over a shared medium whose characteristics can change quickly over time and in an unpredictable manner, and as users are often mobile and associations dynamic. WiFi

Permission to make digital or hard copies of part or all of this work for personal or classroom use is granted without fee provided that copies are not made or distributed for profit or commercial advantage and that copies bear this notice and the full citation on the first page. Copyrights for third-party components of this work must be honored. For all other uses, contact the owner/author(s).

SIGCOMM '15 August 17-21, 2015, London, United Kingdom

(C) 2015 Copyright held by the owner/author(s).

ACM ISBN 978-1-4503-3542-3/15/08.

DOI: http://dx.doi.org/10.1145/2785956.2790037 networks offer several unique knobs to influence the probability of successful transmissions, such as transmission rate and power, as well as retry chains. This introduces opportunities for a finegrained and application specific transmission control, e.g., for service differentiation.

Our proposed architecture, OPENSDWN [2], is based on a unified, programmable control plane which allows to manage both the datapath as well as the virtualized middleboxes. Specifically, OPENSDWN implements per-client virtual access points and perclient virtual middleboxes, to render network functions more flexible and support mobility and seamless migration of network processing functions.

This demo presents interesting use cases of OPENSDWN: (1) OPENSDWN enables service differentiation capabilities, and allows administrators or users to specify application and flow priorities. These priorities are implemented using a fine-grained transmission control. (2) Using its per-client virtual access points and virtual middleboxes, OPENSDWN supports seamless user mobility, as well as flexible function allocation (e.g., function collocation at night to save energy). (3) Network functions such as firewalls and NATs can be deployed flexibly, e.g., outside user premises. (4) OPENSDWN also introduces flexibilities in terms of network control: the system exposes a participatory interface à la [1], where users can indicate priorities for their applications. The control can also be outsourced to an Internet Service Provider (ISP), e.g., for troubleshooting.

\section{THE OPENSDWN SYSTEM}

OPENSDWN is based on an SDN+NFV (a.k.a. $S D N v 2$ ) approach and consists of the following components:

1. Unified Programmability and Abstractions: The logically centralized control plane unifies SDN and NFV through programmatic abstractions. That is, OPENSDWN virtualizes both access points and virtualized middleboxes (see Figure 1(b)), which facilitates an easy handling and migration of per-client state, also beyond CPE boundaries. The OPENSDWN abstractions can be seen as an extension of Odin [3] to NFV: Odin's LVAP concept abstracts the complexities of the IEEE 802.11 protocol stack (client associations, authentication, and handovers), and enables the unified slicing of both the wired and wireless portions of the network by encapsulating the client's Openflow state. OPENSDWN additionally introduces per-client virtual middleboxes, short 


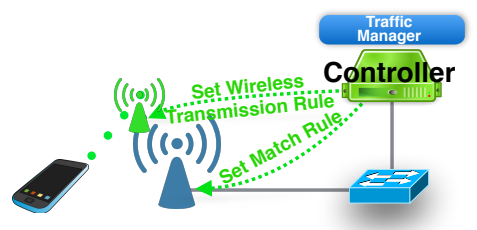

(a) Transmission control: The controller sets specific wireless transmission and OpenFlow rules for per-flow wireless transmission control.

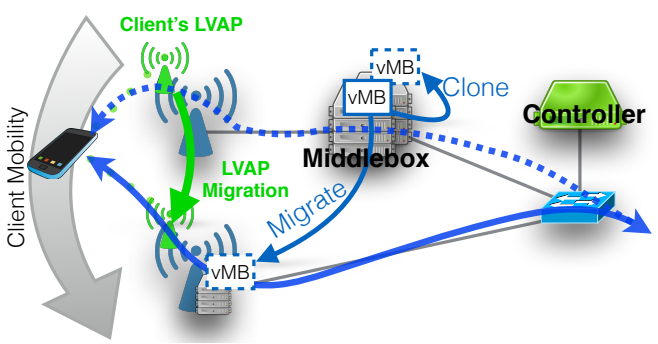

(b) Mobility support: Virtual middleboxes (e.g., encapsulating firewall connection state) can be migrated in the presence of mobility and cloned for redundancy.

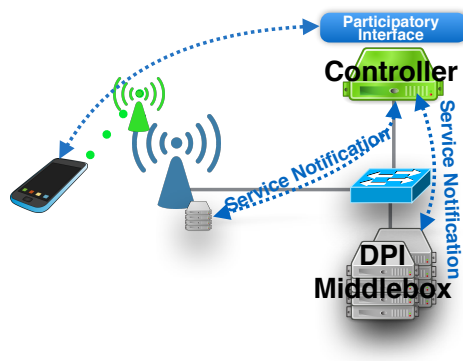

(c) Participatory interface: A participatory application provides an interface to the user. Service detection is achieved through DPI.

Figure 1: Three basic operations supported by OPENSDWN.

$\mathrm{vMB} s$, which can be transferred seamlessly across the network. Specifically, a $v M B$ encapsulates the client's $M B$ state as a virtual $M B$ object. Thus, OPENSDWN achieves control logic isolation as SDN/NFV applications running on top the controller can only operate on their respective LVAPs and $v M B$ s.

2. Programmable Datapath: The programmable datapath allows us to specify per-flow transmission settings, as shown in Figure 1(a). The settings include transmission power, transmission rate as well as tailored retry chains. It is even possible to differentiate between different packets of the same flow (5-tuple): for instance, key frames of a live stream may be given higher priority. This is achieved by using an IDS such as Bro for packet classification and tagging: transmission settings are chosen depending on the tag.

3. Participatory Interface: OPENSDWN's participatory interface allows us to define flow priorities as well as priorities over customers. The chosen priorities are translated by the controller into meaningful network policies. Priorities can be adjusted anytime. Figure 1(c) depicts the participatory interface.

\section{DEMO}

\section{User-Defined Service Differentiation}

OPENSDWN offers visibility into the network's state and supports a fine-grained transmission control, by allowing administrators and users to set per-flow and per-packet specific transmission settings (such as transmission rate, power, retransmission and RTC/CTS strategy). For instance, as we will demonstrate, OPENSDWN can protect latency-sensitive flows (e.g., live streaming) from competing with background traffic (e.g., a Dropbox synchronization). Especially given today's trend to deploy more and more wireless devices in the user's premises, traffic can significantly interfere, e.g., an unimportant system update for a device can easily interfere with requested on demand services such as Spotify or Netflix, resulting in poor performance.

\section{Mobility and migration}

By virtualizing not only the per-client access points, but also the middleboxes, OPENSDWN supports both seamless user mobility and a dynamic resource allocation. The more dynamic resource management introduced by OPENSDWN enables the adjustment and migration of resources and functionality with the user, e.g., for flexibly scaling up or down resources depending on the demand. By collocating network functions, e.g., at night, also energy may be saved. The firewall state migration service is a reactive application triggered through external events to move state between $\mathrm{MB}$ instances. For example, when Odin [3] detects a client with a higher RSSI at a new AP, a handover event is generated and the client's stateful firewall state migrated to the AP before the handover is executed. The firewall state migration service then decides whether the state associated with the mobile user needs to be migrated and executes the operation. The application keeps a mapping between APs and firewalls. If the client is moving over to an AP that corresponds to a different stateful firewall than the current, a migration of the client's flow state is performed.

\section{Smart Direct Multicast Service}

OPENSDWN can also be used in conjunction with group abstractions. Multicast is a standard group communication abstraction, and with the advent of IPv6, the fraction of multicast traffic is likely to grow in the future, e.g., (IPv6 realizes broadcast over multicast and $\mathrm{mDNS}$ to broadcast features to neighboring stations.

In IEEE 802.11, multicasts are typically sent at basic rate. However, wireless networks may benefit from a direct multicast service (DMS) which has the potential of reducing the transmission time over regular multicast, by sending 802.11 packets as unicast. Unfortunately, DMS requires a client to signal its DMS capabilities to the AP, which is the reason why DMS is rarely used in 802.11 networks today.

With OPENSDWN, a controller can detect the number of subscriptions for a particular multicast service and control the transmission accordingly. Specifically, a controller can install an OpenFlow rule to switch from multicast to unicast for the transmission. Moreover, OPENSDWN allows to assign WDTX group-based transmission rules, e.g., transmit a stream of multicast data at the maximum common transmission rate.

Acknowledgments. Research supported by the Federal Ministry of Education and Research (BMBF) Software Campus "SDWN" Project Grant (Reference number 01IS12056).

\section{REFERENCES}

[1] A. D. Ferguson, A. Guha, C. Liang, R. Fonseca, and S. Krishnamurthi. Participatory networking: An api for application control of sdns. SIGCOMM Comput. Commun. Rev., 43(4):327-338, Aug. 2013.

[2] J. Schulz-Zander, C. Mayer, B. Ciobotaru, S. Schmid, and A. Feldmann. OpenSDWN: Programmatic Control over Home and Enterprise WiFi. In SOSR '15.

[3] J. Schulz-Zander, L. Suresh, N. Sarrar, A. Feldmann, T. Hühn, and R. Merz. Programmatic orchestration of wifi networks. In USENIX ATC'14. 